Article

\title{
Antibacterial-Agent-Immobilized Gelatin Hydrogel as a 3D Scaffold for Natural and Bioengineered Tissues
}

\author{
Tuyajargal Iimaa ${ }^{1,2}$, Takaaki Hirayama ${ }^{1}$, Nana Shirakigawa ${ }^{1}{ }^{1}$, Daisuke Imai ${ }^{3}$, \\ Takanobu Yamao ${ }^{4}$, Yo-ichi Yamashita ${ }^{3,4}$, Hideo Baba ${ }^{4}$ and Hiroyuki Ijima ${ }^{1, *(1)}$ \\ 1 Department of Chemical Engineering, Graduate School of Engineering, Kyushu University, 744 Motooka, \\ Nishi-Ku, Fukuoka 819-0395, Japan; tuyajargal@mnums.edu.mn (T.I.); t.r.y.k.t.@@kyudai.jp (T.H.); \\ shirakigawa@chem-eng.kyushu-u.ac.jp (N.S.) \\ 2 Department of Biochemistry and Laboratory Medicine, School of Biomedicine, Mongolian National \\ University of Medical Sciences, Ulaanbaatar 14210, Mongolia \\ 3 Department of Surgery and Science, Graduate School of Medical Sciences, Kyushu University, 3-1-1, \\ Maidashi, Higashi-ku, Fukuoka 812-8582, Japan; dice.m325@gmail.com (D.I.); \\ y-yama@kumamoto-u.ac.jp (Y.-i.Y.) \\ 4 Department of Gastroenterological Surgery, Graduate School of Medical Sciences, Kumamoto University, \\ 1-1-1 Honjo, Chuo-ku, Kumamoto 860-8556, Japan; i_am_kida7172205@yahoo.co.jp (T.Y.); \\ hdobaba@kumamoto-u.ac.jp (H.B.) \\ * Correspondence: ijima@chem-eng.kyushu-u.ac.jp; Tel.: +81-92-802-2748; Fax: +81-92-802-2748
}

Received: 9 May 2019; Accepted: 9 June 2019; Published: 11 June 2019

\begin{abstract}
Hydrogels and their medical applications in tissue engineering have been widely studied due to their three-dimensional network structure, biocompatibility, and cell adhesion. However, the development of an artificial bile duct to replace the recipient's tissue is still desired. Some challenges remain in the tissue engineering field, such as infection due to residual artifacts. In other words, at present, there are no established technologies for bile duct reconstruction as strength and biocompatibility problems. Therefore, this study investigated hydrogel as an artificial bile duct base material that can replace tissue without any risk of infectious diseases. First, an antibacterial agent (ABA), Finibax (an ABA used for the clinical treatment of biliary tract infection), was immobilized in gelatin using a crosslinking agent, and the antibacterial properties of the gel and its sustainability were tested. Furthermore, the immobilized amount and the improvement of the proliferation of the human umbilical vein endothelial cells (HUVECs) were cultured as the ABA-Gelatin hydrogel was introduced to prepare a 3D scaffold. Finally, we performed hematoxylin and eosin (H\&E) staining after subcutaneous implantation in the rat. Overall, the ABA-Gelatin hydrogel was found to be viable for use in hydrogel applications for tissue engineering due to its good bactericidal ability, cell adhesion, and proliferation, as well as having no cytotoxicity to cells.
\end{abstract}

Keywords: hydrogel; antibacterial agent; gelatin; 3D scaffold; biomaterials for tissue engineering; cytotoxicity; cell culture

\section{Introduction}

In general, tissue engineering and regenerative medicine require scaffolds that have a cell adhesion surface and cell growth area [1] and seek to invent desired parts for living organisms [2]. The importance of tissue engineering is demonstrated by the demand for tissue transplantation and regeneration [3]. The application of biopolymer-based hydrogels dates back to 1960 when researchers introduced the use of soft contact lens material [4]. Hydrogels possess flexibility very similar to natural tissue due 
to their large water content, soft consistency, and excellent biocompatibility [5]. They also show a minimal tendency to adsorb proteins from body fluids because of their low interfacial tension [4]. Therefore, in recent years, hydrogels have had many different functions in the field of tissue engineering. In addition, hydrogel scaffolds are being applied to transplant cells and to engineer tissues in the body, including cartilage, liver, and smooth muscle [2].

Biliary complications are a significant cause of morbidity and mortality following orthotopic liver transplantation, with estimates of their incidence ranging between $11.5 \%$ and $34 \%$. In a survey of 30 major transplant centers in the US, biliary complications occurred in $16 \%$ of patients after liver transplantation [6]. Additionally, the post-operative course for an extra-hepatic bile duct is complicated by retrograde infections via the intestine or stenosis at the anastomosis requiring re-anastomosis [7]. There are some reports on an association of bacterial infection with the main disorders of the biliary tract [8]. For this reason, this study aimed to determine the effect of an antibacterial agent with gelatin gel on transplantation in a rat study.

Finibax, as a 1- $\beta$-methyl carbapenem, is a cell-wall synthesis inhibitor. This carbapenem has been activated as both imipenem and meropenem against both gram-positive and gram-negative bacteria. Moreover, these kinds of antibiotic have excellent in vitro activity and pharmacological properties [9]. Particularly, they treat gram-negative bacteria that are resistant to most antibiotics, including carbapenems and cephalosporins. Surveillance of drug resistance has shown that bacteria-expressing $\beta$-lactamases have attracted the attention of modern researchers due to their resistance to a broad range of $\beta$-lactams, including third-generation cephalosporin derivatives [10]. Carbapenemases are $\beta$-lactamases with versatile hydrolytic capacities. Therefore, they can be used for both in vivo and in vitro biomedical applications [11].

As mentioned above, hydrogels have been utilized as scaffold materials for drug delivery and a variety of other applications [12]. This paper focuses on the use of hydrogel as a scaffold for tissues engineering. Therefore, we fabricated a bile duct scaffold using a natural polymer with an antibacterial agent and then transplanted the scaffold into rats in order to investigate the functional potential as a replacement for the native bile duct with its morphology after transplantation. We identified three categories of scaffold applications in this paper-the antibacterial agent, the bioactive molecule delivery, and the cell/tissue delivery. It is expected that this 3D scaffold for transplantation is useful for regenerative medicine based on tissue engineering.

\section{Results and Discussion}

\subsection{Selection of an $A B A$}

In this section of the study, our aim was to develop a scaffolding base material with antibacterial properties, and three antibacterial agents (ABA) were considered as candidates for this study-Finibax, Flomoxef, and Biapenem - as carbapenems and cephalosporins show broad antibacterial activities (Table 1). These ABAs exert antibacterial properties by inhibiting bacterial cell-wall synthesis and lysing. They can bind and inactivate the protein-binding protein, an enzyme for cell-wall synthesis $[13,14]$.

Table 1. Antibacterial agents.

\begin{tabular}{llll}
\hline FDA *-Approved ABA & Trade Name & Category & Approval Year/Trial Year \\
\hline Finibax & Doripenem & $\beta$-Lactam-carbapenem & 2007 \\
Flomoxef & Flumarin & $\beta$-Lactam-cephalosporins & 1988 \\
Biapenem & Omegacin & $\beta$-Lactam-carbapenem & 2002 \\
\hline \multicolumn{4}{c}{$*$ US Food and Drug Administration, (FDA). }
\end{tabular}

The ABA solutions were dropped (Figure $1 \mathrm{a}, \mathrm{b}$ ) into position on a dish and the bactericidal effect against Escherichia coli (E. coli) was confirmed with each ABA (Figure 1c,d,f,g). We selected E. coli as a gram-negative (aerobic and anaerobic growth) bacteria. The antibacterial effect of the agents was more 
pronounced against gram-negative bacteria than gram-positive organisms [9]. Importantly, in a study by Tajeddin et al. a significant association was observed between the colonization of $E$. coli and biliary tract diseases [8].
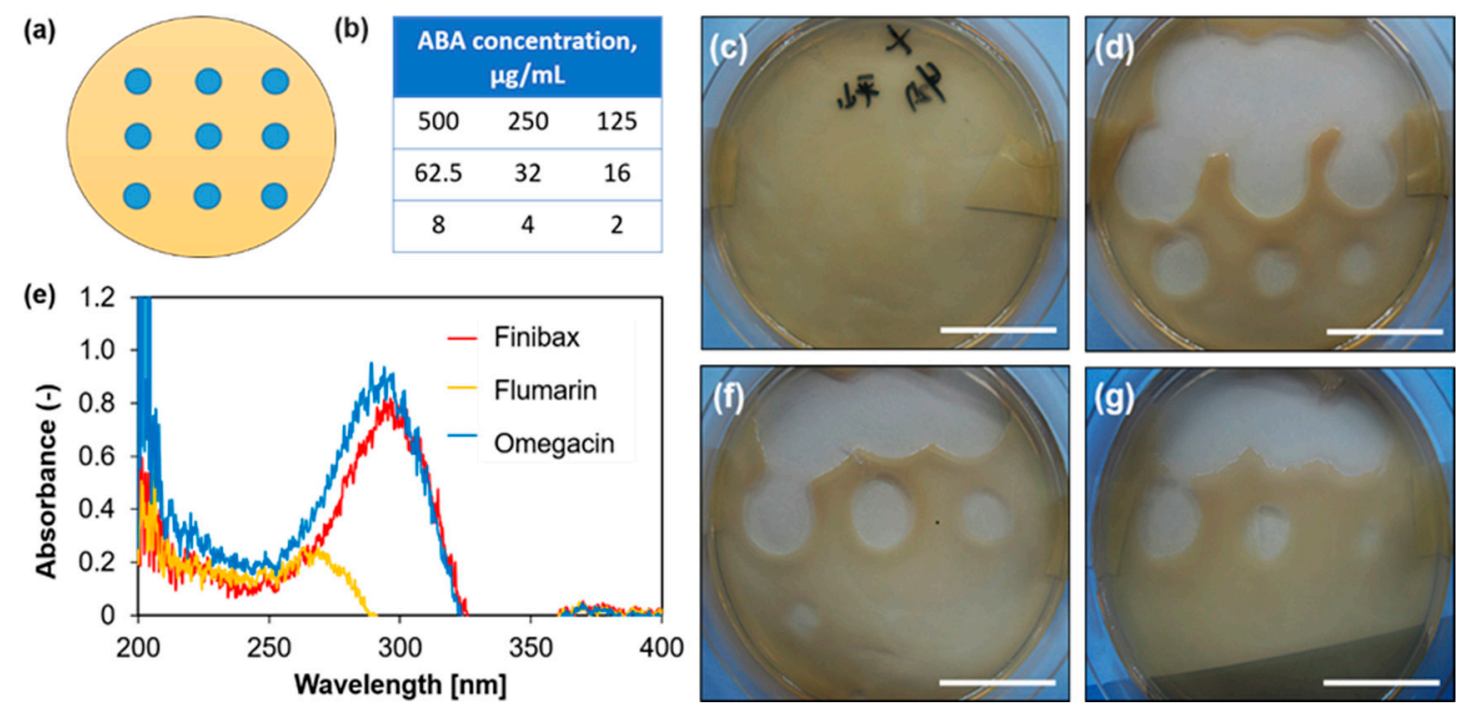

Figure 1. Descriptions of the images of the experiment: (a) The dropped position of the antibacterial agent (ABA) solutions in a dish; (b) the ABA concentrations: $2-500 \mu \mathrm{g} / \mathrm{mL}$ on the point; and (e) the spectral wavelength of the antibacterial agents, namely Finibax, Flomoxef, and Biapenem. Photographs of the action of the ABAs in a dish of transformed Escherichia coli: (c) control or ABA-free; (d) Finibax; (f) Flomoxef; and (g) Biapenem after 1 day of the inoculation. The bar represents $30 \mathrm{~mm}$.

First of all, we evaluated what concentration range of ABA exhibits the antibacterial effect. The effects of Finibax, Flomoxef, and Biapenem with E. coli were determined at around $2.0 \mu \mathrm{g} / \mathrm{mL}$, $8.0 \mu \mathrm{g} / \mathrm{mL}$, and $16 \mu \mathrm{g} / \mathrm{mL}$ or more, respectively. This indicated that each ABA concentration showed different effects in several parts.

For spectral analysis, the maximum absorption wavelengths of Finibax and Biapenem were found at approximately $300 \mathrm{~nm}$, while Flomoxef was found at $260 \mathrm{~nm}$ (Figure 1e). Additionally, by using a calibration curve of absorbance and concentration at that peak, we found that it was possible to easily measure the concentration of the ABA in the sample.

The phenomena suggest that the higher concentration of each ABA apparently enhanced the antibacterial effect (Figure 1c,d,f,g). In other words, it would be possible to prepare a scaffolding base material with antibacterial properties if the ABA could be contained at a concentration higher than these sufficient values.

The antibacterial activity results clearly showed that this study should use Finibax, which showed its effect at a low concentration and had a large peak wavelength. In addition, hydrogels that contain $\beta$-lactam drug inhibitors, such as carbapenems, e.g., doripenem (Finibax), and cephalosporins, e.g., cefotaxime and ceftazidime [15], are used in urinary catheters, can prevent bacterial colonization on the surface, and provide a smooth and slippery surface to improve its biocompatibility [16]. Additionally, we considered the fact that Finibax is actually used for treatment by interfering in the synthesis of the bacterial cell-wall [15].

\subsection{Immobilization of the $A B A$}

We tried to immobilize the ABA as antibacterial sustainability can be improved by crosslinking an ABA with gelatin. The hydrogel removed from a 48-well plate is shown in Figure 2a. The crosslinking was carried out using 1-Ethyl-3-(3-dimethylaminopropyl)-carbodiimide (EDC)/ $N$-hydroxysuccinimide (NHS). This agent reacted with the ABA and gelatin, which both have functional groups. $\beta$-lactams, as shown in Figure 2b, have a four-membered lactam ring [15], while gelatin is a heterogeneous mixture 
of single- or multi-stranded polypeptides, each with extended left-handed proline helix conformations and contain between 50 and 1000 amino acids [17].
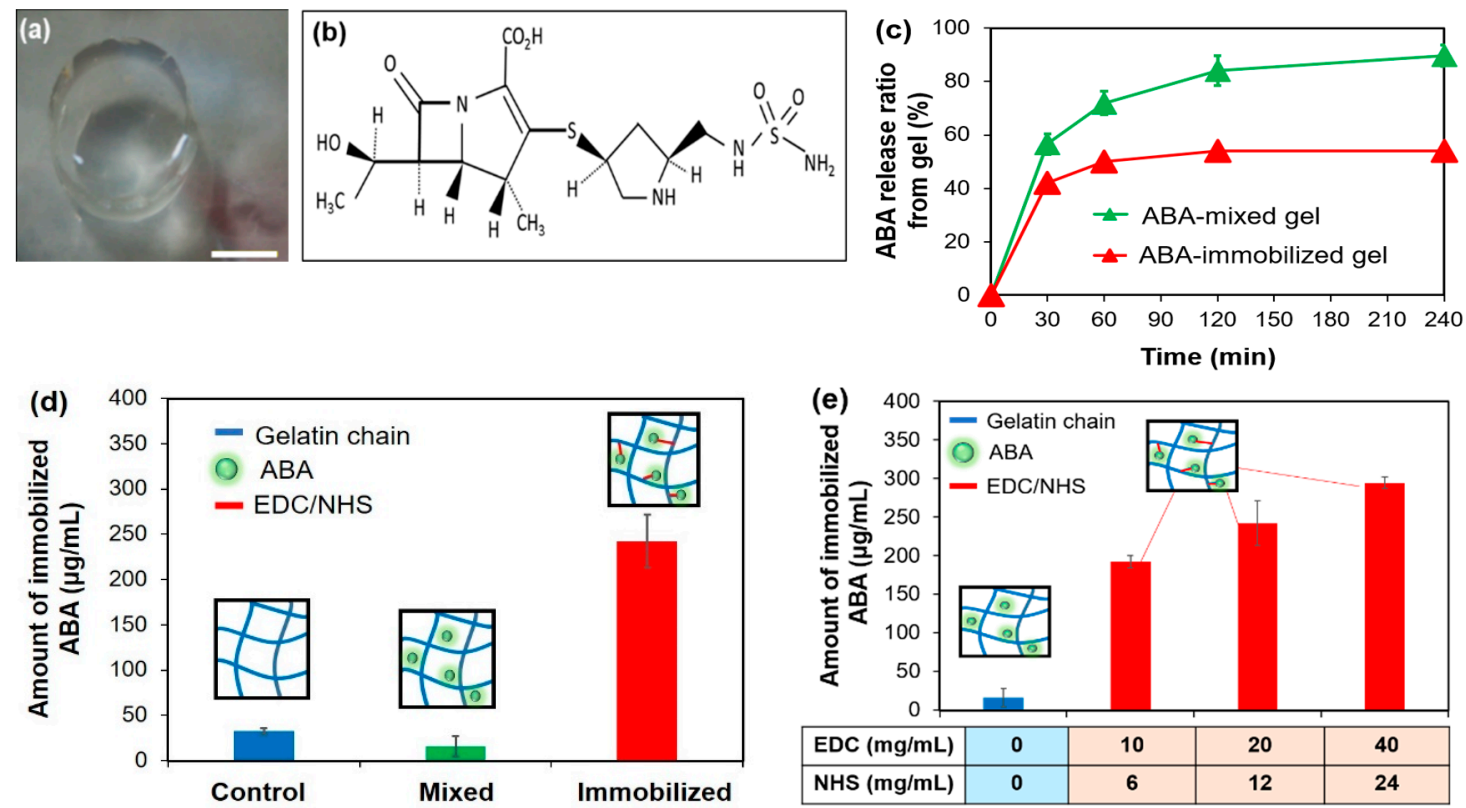

Figure 2. (a) Photograph of the ABA-Gelatin gel- the bar represents $5 \mathrm{~mm}$; (b) the molecular structure of Finibax as an ABA; (c) the release percent of ABA in the gels; (d) the immobilized ABA amount in the ABA-free, ABA-mixed, and ABA-immobilized gelatins; and (e) the immobilized ABA amount at different concentrations of the crosslinking agent.

Hydrogels do not disintegrate during swelling, thanks to their crosslinked structure $[16,18]$. The chemical crosslinking covered here involved the grafting of monomers on the backbone of the polymers or the use of a crosslinking agent to link two polymer chains through their functional groups (such as $\mathrm{OH}, \mathrm{COOH}$, and $\mathrm{NH}_{2}$ ) with a crosslinker, such as EDC [19]. EDC is a zero-length crosslinking agent used to couple carboxyl or phosphate groups to primary amines.

To increase the stability of this active ester, NHS or N-hydroxysulfoxuccinimide (sulfo-NHS) can be used. One of the main advantages of EDC/NHS coupling is water solubility, which allows direct bioconjugation to be carried out under physiological conditions [20]. In addition, using the EDC/NHS coupling, almost all kinds of molecules (e.g., enzymes, antibodies, peptides, DNA, and fluorophores) can be attached to the surface without prior modification [21].

Figure $2 \mathrm{c}$ shows the ratio of the released ABA from the gels using a calibration curve. About $90 \%$ and $50 \%$ of the ABA was released slowly in the ABA-mixed gelatin gel and the ABA-immobilized gelatin gel, respectively. This result suggested that the ABA was successfully immobilized in gelatin by the EDC/NHS. From the release study, we found that the ABA treated with the gel was immobilized as well as released slowly.

Next, in order to measure the amount of immobilized ABA, an evaluation was made by decomposing the gel. From the calibration curve, the ABA concentration of the decomposing solution was detected-the results are shown in Figure 2d. By comparing the mixing condition with the gelatin only, the equivalent results were obtained. It was assumed that the unfixed ABA was washed out. While, in the immobilized condition, the concentration was significantly greater than that of the other two conditions. It was confirmed as well that the ABA was immobilized by the EDC/NHS coupling.

Additionally, we compared the crosslinking potential of the EDC/NHS couples in different molar ratios (Table 2). Our experimental results showed that the immobilized density in the gel could be manipulated by a crosslinking agent-the higher concentration of the EDC/NHS apparently enhanced 
the immobilized density of the ABA (Figure 2e). From this result, we focused on the second component that contains $20 \mathrm{mg} / \mathrm{mL}$ EDC:12 mg/mL NHS for future studies. This coupling can be immobilized, $250 \mu \mathrm{g} / \mathrm{mL}$ ABA is the effective value for Finibax (Figure 1d).

Table 2. The molar ratio of the crosslinking couple.

\begin{tabular}{lll}
\hline \multirow{2}{*}{ Case No. } & Concentration, $\mathbf{m g} / \mathbf{m L}$ & \\
\cline { 2 - 3 } & EDC & NHS \\
\hline 1 & 0 & 0 \\
2 & 10 & 6 \\
3 & 20 & 12 \\
4 & 40 & 24 \\
\hline
\end{tabular}

\subsection{Evaluation of the Antibacterial Activity of the ABA after Immobilization}

In the previous experiment result, it was suggested that the ABA was immobilized to the gelatin gel. However, it was necessary to evaluate the antibacterial property of the ABA after immobilization.

E. coli activity was measured by the appearance of turbidity. Turbidity is generally used as an indicator of the number of bacteria [22]. Therefore, the correlation was investigated from the calibration curve of $E$. coli. The response of $E$. coli liquid was found to be linear in the investigated concentration range as shown in Figure 3a. The correlation coefficient was represented by the linear association between the bacteria number and the turbidity. It can be seen from the test results that the calibration curves for $E$. coli were correlative within a range of CFU/mL. From the counting result, the number of coliform bacteria in the E. coli liquid was calculated. The correlation between the number of E. coli and the turbidity was obtained. It was shown that the turbidity increased as the number of E. coli increased.
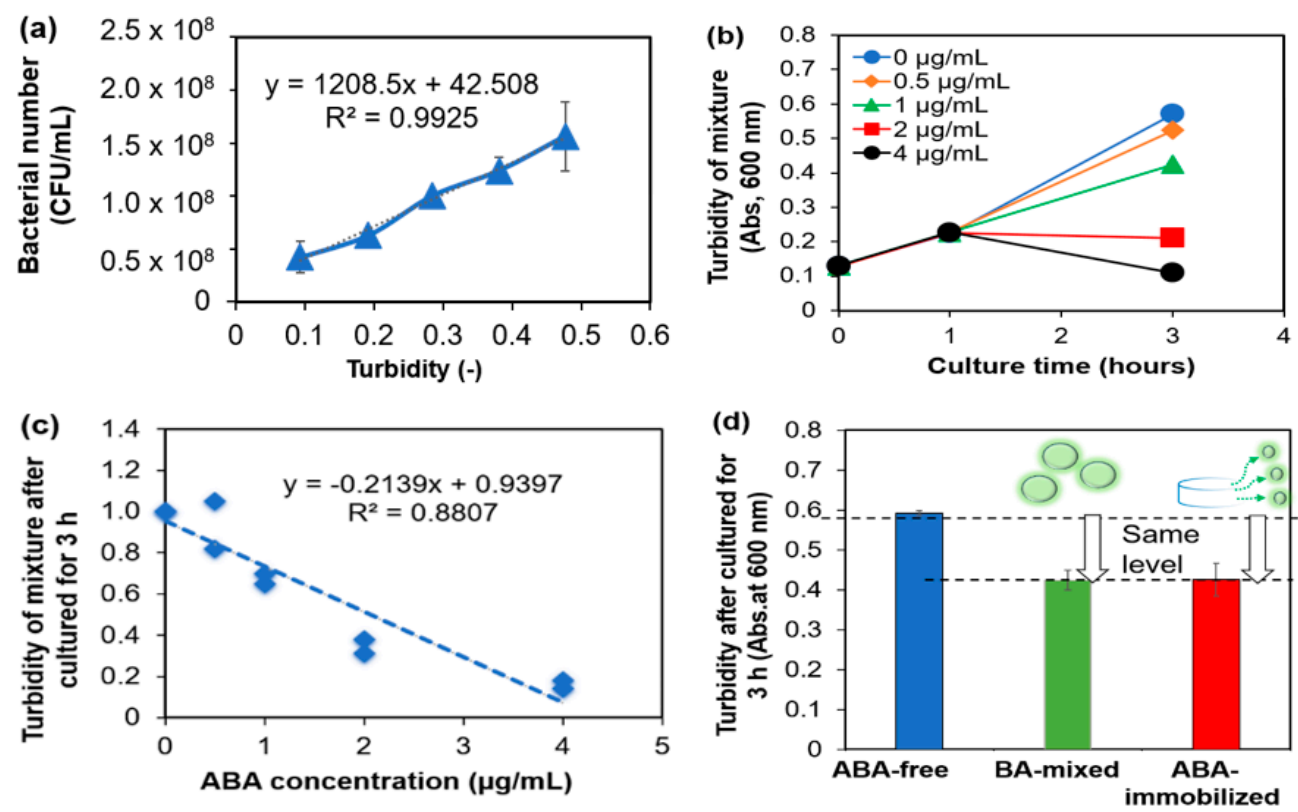

Figure 3. (a) Calibration curve of the number of E. coli (CFU/mL) and the turbidity value; (b) temporal change of the turbidity at different concentrations of the ABA; (c) calibration curve of the turbidity and the concentration of the ABA after three hours; and (d) evaluation of the activity of the ABA in free, mixed, and immobilized cases.

As an antibacterial property of the solution, the turbidity was investigated for $3 \mathrm{~h}$ by mixing the E. coli liquid and the ABA solution in different concentrations. The changes in the turbidity over time at each ABA concentration are shown in Figure 3b. The turbidity results had the same values at all concentrations within $1 \mathrm{~h}$. It was clear that after $1 \mathrm{~h}$ of mixing, the ABA concentrations were affected 
differently. Therefore, after $3 \mathrm{~h}$, it was confirmed that the turbidity became lower as the concentration of the ABA increased. Our experimental results showed that the antibacterial activity improved as the concentration became higher.

Furthermore, to investigate the antibacterial properties of the unknown specimen, the objective of this study was to prepare a calibration curve (Figure 3c). On the contrary, the result showed a good correlation for E. coli, with a correlation coefficient $\left(\mathrm{r}^{2}\right)$ of approximately 0.88 , showing a high correlation. Mainly, it was suggested that the antibacterial property of unknown specimens can be measured using the calibration curve.

The amide bond is formed then between the ABA and the gelatin with crosslinking, the structure of the ABA is changed, and then the ABA may be inactivated. Therefore, to determine the activity of the ABA, we cultured E. coli in gel digestion solution. The ABA-free, the ABA without the immobilization, and the ABA with the immobilization were mixed with the E. coli liquid and cultured (Table 3). After 3 $h$ of the culture, the turbidity (an indicator of the number of bacteria) was examined and the activities of the ABA were compared. The turbidity after $3 \mathrm{~h}$ of incubation is shown in Figure $3 \mathrm{~d}$. From the previous study, it was confirmed that the turbidity after $3 \mathrm{~h}$ of E. coli culture depended on the ABA concentration. The decomposed solution of the ABA-immobilized gelatin gel was mixed with E. coli, and the turbidity was measured at about 0.4 . Similarly, the turbidity of ABA mixed with E. coli also showed a value of about 0.4 . On the other hand, the same turbidity was detected in these solutions by spectral measurement. It was suggested that the gel digestion liquid had an equivalent antibacterial activity to the ABA concentration. Overall, after immobilization, the activity of the ABA was the same as before immobilization.

Table 3. Composition of the gels.

\begin{tabular}{ll}
\hline Condition Name & Composition \\
\hline ABA-free & Collagenase solution mixed with E. coli liquid \\
Non-immobilized ABA & ABA solution (solvent: collagenase solution) mixed with E. coli liquid \\
Immobilized ABA & ABA-immobilized gel mixed with E. coli liquid \\
\hline
\end{tabular}

\subsection{Antibacterial Activity of the Gel}

To observe the activity of the ABA on the E. coli growth, the ABA-free gelatin gel and the ABA-mixed gelatin gel were placed on agar medium, the results are the images in Figure 4a,b. One day after the inoculation with E. coli, the whole agar medium appeared yellow. However, the agar medium became transparent in the ABA-mixed gelatin gel, and the growth suppression of E. coli was confirmed. It was visually confirmed that the ABA was gradually released from the gel and that the E. coli growth decreased.

In addition, the number of bacteria on the gel surface 1 day after the culture was calculated from the number of colonies (Figure $4 \mathrm{c}, \mathrm{d}$ ). The doubling time was calculated from the number of bacteria at sowing: PBS was $89 \mathrm{~min}$, while the LB medium was $62 \mathrm{~min}$. In the LB medium, the doubling time was shorter because of the abundance of amino acids and minerals. Colonies were formed in both the PBS and LB mediums and were solvent under the condition without ABA. The bacterial number on the gel surface increased from 539 (on day 0) to $2.32 \times 10^{6}$ in PBS and $1.01 \times 10^{8}$ in LB medium (on day 1). In contrast, under the ABA immobilization conditions, the formation of colonies was not confirmed. This suggests the antimicrobial property of the ABA-immobilized gelatin means that bacteria do not grow on the surface upon transplantation and infection is prevented.

The antimicrobial property of the ABA-immobilized gelatin was confirmed in vitro. However, sometimes a surgical site infection occurs after surgery in vivo. For this reason, the gels were transplanted subcutaneously, the bacteria adhering to the gels was investigated, and the antibacterial property was evaluated in vivo. We measured the number of bacteria in rat subcutaneous implantation. No colonies of bacteria were confirmed in both the ABA-free and ABA-immobilized conditions (Figure 4e,f). This result suggested that there were no subcutaneous bacteria, so antibacterial activity under the skin could not be evaluated. On the other hand, in a clinical investigation, it is necessary 
to transplant into the abdominal cavity where there are organs, such as the intestines, and evaluate antibacterial activity.
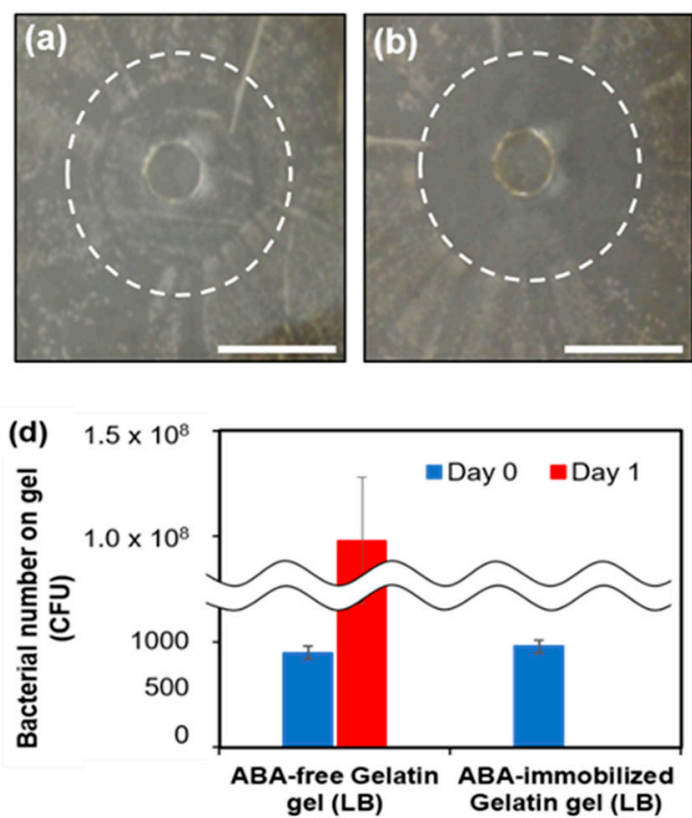
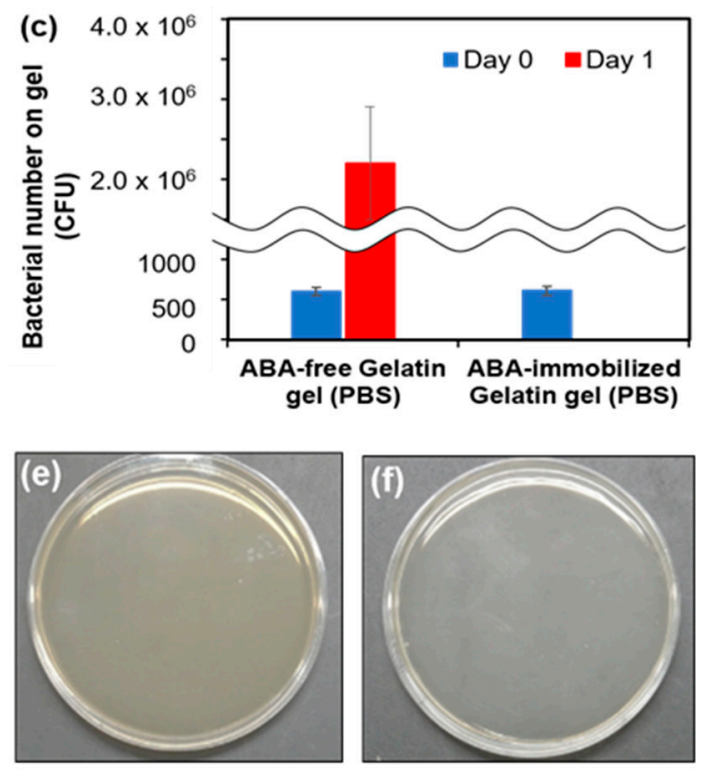

Figure 4. Photographs of the antibacterial evaluation on E. coli: (a) ABA-free gelatin gel and (b) ABA-immobilized gelatin gel. The bars represent $1 \mathrm{~cm}$. Measurements of bacterial count on the gel surface: (c) PBS and (d) LB medium. Agar medium coated with PBS immersed in gel: (e) Gelatin without an antibacterial agent and (f) gelatin mixed with an antibacterial agent.

Moreover, turbidity measurements were used to determine the increase in the ratio of turbidity for different gels. The growth ratio was calculated by dividing the turbidity and the initial turbidity (Figure 5a). In the ABA-free gel condition, the turbidity increased from the first time, and thereafter it hardly changed. The difference between the first and the second case was considered to be due to the first decrease in the turbidity rate by the EDC/NHS remaining in the gel. In contrast, under the immobilization condition, the turbidity did not increase at all until the third time, while it increased greatly after the fourth time. A possible reason for this increase is that the ABA, which had not been immobilized flowed out due to the gel being used without washing and the effect was exhibited. Although it increased after the fourth time, the turbidity rate was lower than in the ABA-free condition, and the growth of the bacteria could be inhibited. From the above, it is suggested that antibacterial properties are observed near the ABA-immobilized gelatin gel.
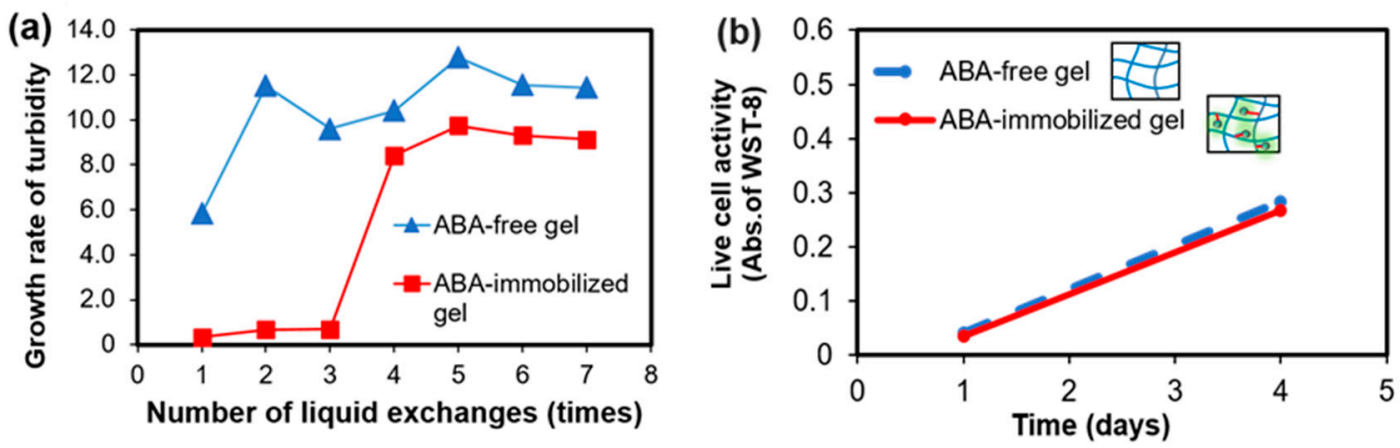

Figure 5. ABA-free gelatin gel and ABA-immobilized gelatin gel: (a) Turbidity increase rate with respect to the number of liquid changes and $(\mathbf{b})$ evaluation of the number of viable cells in the human umbilical vein endothelial cells (HUVEC) culture on the gels. 


\subsection{Evaluation of Cytotoxicity of the Gel Surface}

The tissue-engineered artificial bile duct is a combination of three elements, namely a cell, a scaffold, and a growth factor, and is a substitute for living tissue [1,23]. In recent years, hydrogel scaffolds are broadly used for cell delivery and tissue development as they have a highly hydrated 3D network, cell adhesion, proliferation, and differentiation as well as good chemical and mechanical signals to cells [23]. Therefore, hydrogels can be strategized for different applications: (i) to achieve a sustained release with minimum toxicity to the tissues and (ii) to form a responsive surface that possesses antibacterial activity and a self-cleaning ability for long-term usage [24].

Therefore, it is desirable that the scaffold shows no toxicity to cells. In order to investigate how the ABA-immobilized gelatin gel cultured on cells, human umbilical vein endothelial cells (HUVECs), which are a kind of epithelial cells, were selected. By comparing the immobilizing ABA-gelatin condition with the ABA-free condition, almost the same degree of cell proliferation was confirmed, as shown in Figure 5 b. In addition, mitochondrial activity was evaluated by WST- 8 assay, and cell morphology was observed (Figure $6 \mathrm{a}-\mathrm{d}$ ). These results suggested that there was no cytotoxicity by immobilizing the ABA and that the cell proliferation was good. Generally, the hydrophilic surfaces possess low interfacial free energy. Therefore, when in contact with body fluids, hydrogels have good biocompatibility and a low tendency to adhere cells on the gel surface [19]. Furthermore, we performed a visual evaluation by hematoxylin and eosin (H\&E) staining the gel after its subcutaneous implantation in rats.
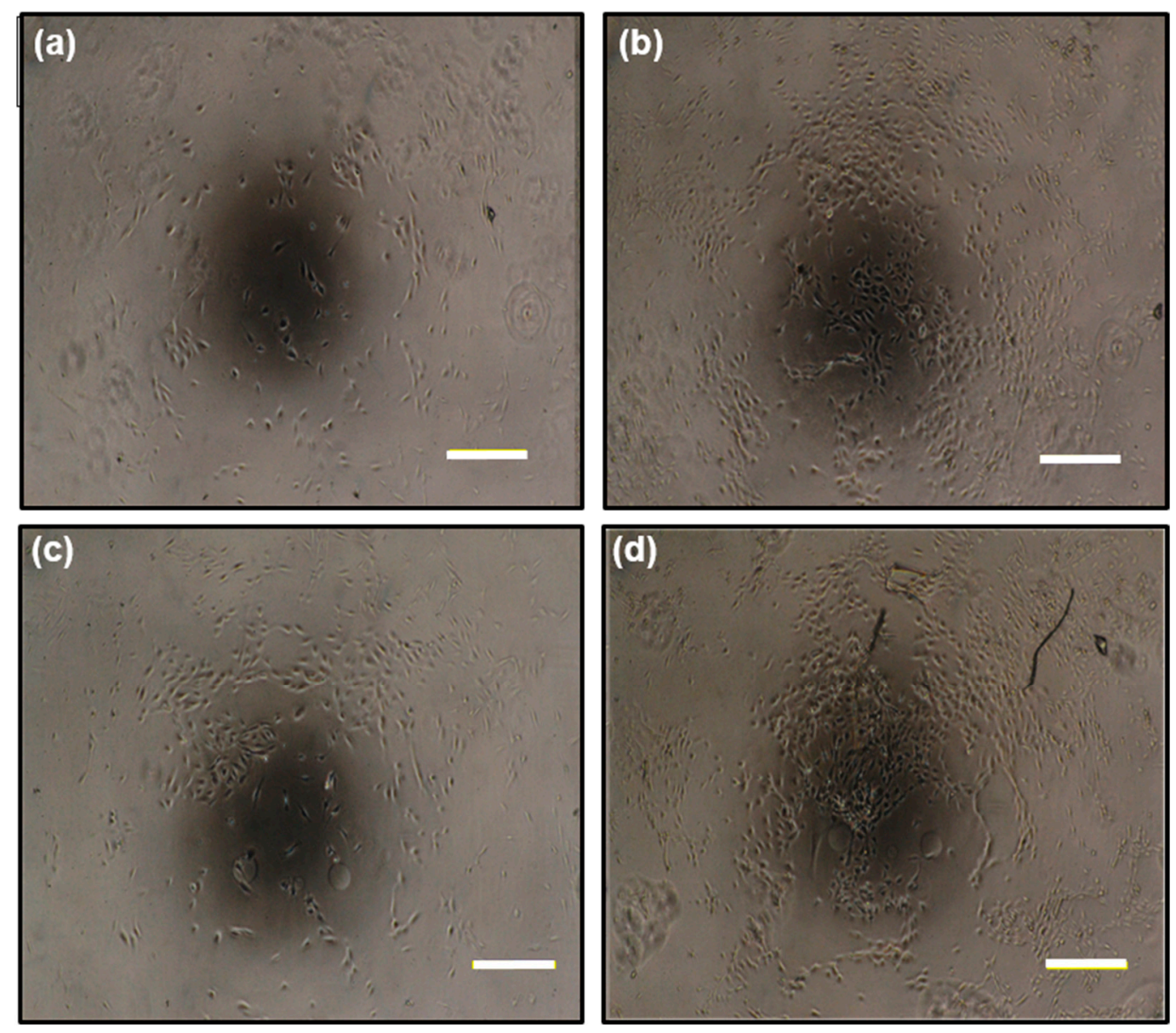

Figure 6. Cell photographs of the human umbilical vein endothelial cell (HUVEC) culture on gelatin gels: 1 day after seeding with (a) an ABA-free gelatin gel and (b) an ABA-immobilized gelatin gel; 4 days after seeding with (c) an ABA-free gelatin gel and (d) an ABA-immobilized gelatin gel. The bars represent $200 \mu \mathrm{m}$. 
In order to investigate how the ABA affected the tissue, the ABA-immobilized gelatin gel was subcutaneously transplanted on the back of a rat, taken out 1 week later, and stained with the H\&E staining. Photographs of the gel after extraction are shown in Figure $7 \mathrm{a}, \mathrm{d}$. One week after the transplantation (Figure $7 \mathrm{~b}, \mathrm{e}$ ), the gel was taken out of the tissue and was expected to have biocompatibility. Results of the H\&E staining are shown in Figure 7c,f. The results of the H\&E staining showed that cells were adhering on the surface of the gels under both conditions, and the gelatin gel was expected to be biocompatible. In addition, some of the color was thickened with an ABA-immobilized gelatin gel, and mild inflammation occurred. However, mild inflammation secretes various cytokines in vivo, including substances that promote tissue replacement. As a clinical study, it is necessary to evaluate this by conducting a long-term transplantation.
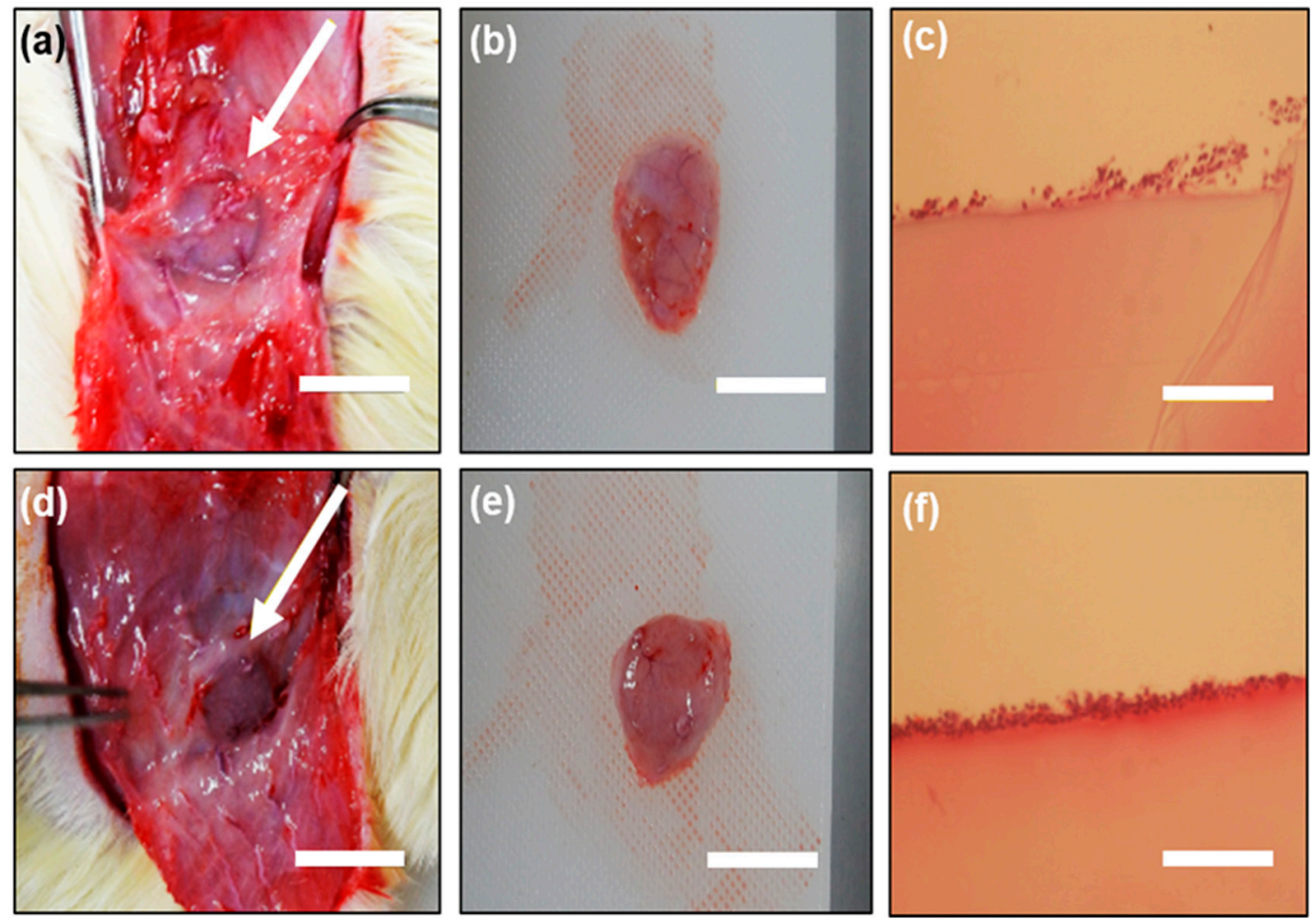

Figure 7. ABA-free gelatin gel: (a) 1 week after transplantation; (b) after the extraction of the gel and (c) hematoxylin and eosin (H\&E) staining of the gel after subcutaneous implantation for 1 week in the rat; ABA-immobilized gelatin gel: (d) 1 week after transplantation; (e) after the extraction of the gel and (f) H\&E staining of the gel after subcutaneous implantation for 1 week in the rat. Bars represent 1 $\mathrm{cm}$ and the arrows indicate the gel sample.

Consequently, many materials have been used for one vital requirement as cells can easily be mixed with the gel. We were able to immobilize the ABA in the gelatin hydrogel for this reason. In conclusion, the ABA-immobilized gelatin hydrogel showed good antibacterial activity and low cytotoxicity both in vitro and in vivo.

\section{Materials and Methods}

\subsection{Chemicals and Reagents}

The materials and reagents in this study were obtained from the following sources: Gelatin from porcine skin (gel strength 300 Type A) was purchased from Sigma Aldrich Ltd (St. Louis, MO, USA); Doripenem and Flumarin were purchased from Shionogi \& Co., Ltd (Osaka, Japan); Omegacin, Streptomycin sulfate, and Penicillin G potassium were purchased from Meiji Seika Pharma Cooperation 
Ltd (Tokyo, Japan); human umbilical vein endothelial cells (HUVECs) were obtained from Kurabo Industries Ltd (Osaka, Japan); collagenase, 2-(N-morpholino)ethanesulfonic acid (MES), D (+) glucose, agar, $N$-hydroxysuccinimide, HEPES, and 10\% formalin were purchased from Wako Pure Chemical Cooperation (Osaka, Japan); Bacto tryptone, a pancreatic digest of casein, was purchased from Beston, Dickinson, and Company (Durham, NC, USA); Bacto yeast was purchased from Sigma Aldrich Co., Ltd (St. Louis, MO, USA); Fasting Blood Sugar (FBS) was purchased from Capricorn Scientific GmbH (Ebsdorfergrund, Germany); EGM-2 was from Lonza Walkersville MD (Walkersvile, MD, USA), and the WST-8 assay kit was obtained from Dojindo (Kumamoto, Japan). All other chemicals were of an analytical grade. Nine-week-old SD rats were obtained from SLC., Co., Ltd (Fukuoka, Japan).

\subsection{Selection of the Antibacterial Agent}

In the evaluation method, E. coli was seeded on an agar medium and the ABA solution at each concentration was dropped onto it. The concentration of the ABA in the solution was measured by the absorbance, then the peak wavelength was determined. The ABAs that were selected in this study are shown in Table 1.

\subsubsection{Evaluation of Effective Concentration}

ABA aqueous solutions of $2-500 \mu \mathrm{g} / \mathrm{mL}$ were prepared. Agar medium $(20 \mathrm{~mL})$ was poured into $90 \mathrm{~mm}$ dishes, and allowed to stand at room temperature until solidified. E. coli was pre-cultured in a shaker at $37^{\circ} \mathrm{C}$ for 1 day. This E. coli was spread on the agar medium. Thereafter, $10 \mu \mathrm{L}$ of each ABA solution was added drop by drop. Cultivation was carried out in an incubator at $37^{\circ} \mathrm{C}$ for 1 day, and the degree of proliferation was observed.

\subsubsection{Spectrum Measurement}

A $50 \mu \mathrm{g} / \mathrm{mL}$ aqueous solution of each ABA—Finibax, Flomoxef, and Biapenem-was prepared, and the absorbance of $100 \mu \mathrm{L}$ of the solutions was measured using an ultraviolet-visible light spectrophotometer (UV-2500 PC, Shimadzu Corporation, Kyoto, Japan).

\subsection{Immobilization of the $A B A$}

\subsubsection{Evaluation Based on the Release of the ABA into the Liquid}

The solution was obtained from the mixture containing $200 \mathrm{mg} / \mathrm{mL}$ of gelatin and $10 \mathrm{mg} / \mathrm{mL}$ of $\mathrm{ABA}$ in a ratio of 1:1. The mixture was poured into a 48 -well plate at $200 \mu \mathrm{L} / \mathrm{well}$, and the gel was obtained after cooling at $4{ }^{\circ} \mathrm{C}$ for $15 \mathrm{~min}$. Next, $200 \mu \mathrm{L} /$ well of EDC/NHS $(20 \mathrm{mg} / \mathrm{mL}: 12 \mathrm{mg} / \mathrm{mL})$ solution was dropped. It was allowed to stand at $4{ }^{\circ} \mathrm{C}$ for $1 \mathrm{~h}$. After the preparation process, the gel was transferred to a 12-well plate, and $2 \mathrm{~mL} /$ well of ultrapure water was added. The absorbance of $100 \mu \mathrm{L}$ of this solution was measured at $300 \mathrm{~nm}$. From the calibration curve of the absorbance and concentration of each ABA, the amount of ABA that flowed out into ultrapure water was calculated by the following formula:

$A B A$ immobilized amount $=$ the $A B A$ concentration in decomposition solution $\times$ liquid volume

\subsubsection{Evaluation of the Immobilization amount by Gel Decomposition}

The preparation method of the gels was as described in Section 3.3.1. The gels were then transferred to $50 \mathrm{~mL}$ centrifuge tubes. An amount of $20 \mathrm{~mL}$ of $0.05 \mathrm{M}$ of 2-( $N$-morpholino)ethanesulfonic acid (MES) buffer was added drop by drop and gently shaken for $24 \mathrm{~h}$. After the supernatants were removed, the same procedure was repeated with $20 \mathrm{~mL}$ of $5 \mathrm{M}$ of $\mathrm{NaCl}$, and $20 \mathrm{~mL}$ of $0.05 \mathrm{M} \mathrm{MES}$ buffer. After washing, the gels were transferred to a 12-well plate, and $2 \mathrm{~mL} /$ well of $0.05 \%$ collagenase solution was added. The solutions were gently shaken at $37^{\circ} \mathrm{C}$ for $3 \mathrm{~h}$. The absorbance of the decomposition solution was measured at $300 \mathrm{~nm}$. 


\subsubsection{Evaluation of the Crosslinking Potential with the EDC/NHS Couple}

The preparation method of the gels was as described in Section 3.3.1. Briefly, the ABA-Gelatin (10 mg/mL:200 mg/mL, 1:1) mixture was added to $200 \mu \mathrm{L}$ of EDC/NHS solutions (Table 2) after cooling at $4{ }^{\circ} \mathrm{C}$ for $15 \mathrm{~min}$. It was allowed to stand at $4{ }^{\circ} \mathrm{C}$ for $1 \mathrm{~h}$ and transferred to a 12 -well plate. Then, $2 \mathrm{~mL} /$ well of ultrapure water was added. The absorbance was measured at $300 \mathrm{~nm}$, and the amount of immobilized ABA was calculated.

\subsection{Evaluation of Antibacterial ( $A B)$ Activity of ABA after Immobilization}

\subsubsection{Correlation Evaluation of the E. coli and Turbidity}

Frozen E. coli liquid was thawed at room temperature. The E. coli liquid and the LB medium were mixed at a ratio of 2:5. The mixture was shaken at $100 \mathrm{rpm}$ in a water bath of $37^{\circ} \mathrm{C}$ for 1 day. Each sample was diluted 100 -fold, and the bacteria number was counted using a counter.

\subsubsection{Correlation Evaluation of the ABA Concentration and Turbidity}

The mixture solution containing the E. coli liquid and the LB medium was described previously in Section 3.4.1. The absorbance of the shaken E. coli liquid was measured at $600 \mathrm{~nm}$. After the LB medium was mixed, the turbidity became 0.145 . The E. coli liquid and the ABA solution of each concentration (solvent: collagenase solution) were mixed at a ratio of 9:1. The mixtures were shaken at $100 \mathrm{rpm}$ in a water bath of $37^{\circ} \mathrm{C}$ for $24 \mathrm{~h}$. An amount of $100 \mu \mathrm{L}$ of each mixed solution was collected at $0 \mathrm{~h}, 1 \mathrm{~h}$, and $3 \mathrm{~h}$. Additionally, the absorbance was measured at $600 \mathrm{~nm}$ while the LB medium was a blank solution.

\subsubsection{E. coli Culture on the Gel}

The preparation method of the gels was as described in Section 3.3.1. The gelatin gels (Table 3) were taken out and poured into a 12-well plate. An amount of 0.05 M MES buffer was added at $2 \mathrm{~mL} /$ well drop by drop. The gels were then transferred to a 6-well plate, and $10 \mathrm{~mL} /$ well of $0.05 \mathrm{M}$ MES buffer was dropped. After the supernatant was removed, $4 \mathrm{~mL} /$ well of $0.5 \%$ collagenase solution was added. Shaking was then carried out on a shaker under conditions of $200 \mathrm{rpm}$ and $37^{\circ} \mathrm{C}$. After 0 $\mathrm{h}, 0.5 \mathrm{~h}, 1 \mathrm{~h}, 1.5 \mathrm{~h}, 2 \mathrm{~h}$, and $24 \mathrm{~h}, 100 \mu \mathrm{L}$ of the supernatant was collected and the absorbance of the ABA was measured at $300 \mathrm{~nm}$ using a blank. This solution was mixed with E. coli liquid at a ratio of 1:9. After mixing for $3 \mathrm{~h}$, the turbidity was measured at $600 \mathrm{~nm}$ and calibrated to $100 \%$ transmittance with $0.5 \%$ collagenase solution.

\subsection{Antibacterial Activity of the Gel Surface}

\subsubsection{Observation of Double Zone Growth Inhibition}

The preparation method of the gels was as described in Section 3.3.1. A phosphate-buffered saline (PBS) solution (-) was dropped at $1 \mathrm{~mL} /$ well, and the gels were left at $4{ }^{\circ} \mathrm{C}$. E. coli liquid (turbidity 0.1 ) was diluted 10-fold and applied to the whole agar medium. Next, the liquid was rinsed with water and incubated at $37^{\circ} \mathrm{C}$.

\subsubsection{E. coli Culture on the Gel}

The preparation method of the gels was as described in Section 3.3.1. $1 \mathrm{~mL} /$ well of PBS (-) and 1 $\mathrm{mL} /$ well of LB medium were each added. The plate was allowed to stand at $4{ }^{\circ} \mathrm{C}$, then $20 \mu \mathrm{L}$ of $E$. coli liquid was dropped. After 1 week of culture, $1 \mathrm{~mL}$ of PBS (-) solution was added, the supernatants were removed, and the remaining liquid was diluted 1000-fold and applied to the agar medium. The number of colonies was measured after 1 day. 


\subsubsection{Measurement of the Number of Bacteria in Rat Subcutaneous Implantation}

The preparation method of the gels was as described in Section 3.3.1. PBS (-) solution was dropped and left at $4{ }^{\circ} \mathrm{C}$. The gels were transplanted subcutaneously into nine-week-old rats. One week after the transplantation, the gels were excised and immersed in $1 \mathrm{~mL}$ of PBS solution. After 1 hour of PBS immersion and soaking, the gels were applied to the agar medium. The agar medium was cultured at $37^{\circ} \mathrm{C}$ for 1 day, and the number of colonies was measured.

\subsubsection{E. coli Bacterial Density in Relation to the Turbidity Method.}

The preparation method of the gels was as described in Section 3.3.1. E. coli liquid was dropped at $2 \mathrm{~mL} /$ well. Turbidity was measured after culturing for $3 \mathrm{~h}$ under a $37^{\circ} \mathrm{C}$ condition. The supernatant was removed, and again E. coli liquid was dropped. After $3 \mathrm{~h}$, the turbidity was measured, and the process was repeated 7 times.

\subsection{Evaluation of the Cytotoxicity of the Gel}

\subsubsection{HUVEC Culture on the Gel}

The preparation method of the gels was as described in Section 3.3.1. Then, $1 \mathrm{~mL}$ of Dulbecco's Modified Eagle Medium (DMEM) with fasting blood sugar (FBS 2\%) was dropped and allowed to stand at $4{ }^{\circ} \mathrm{C}$ for $6 \mathrm{~h}$. After the supernatant was removed, $1 \mathrm{~mL}$ of DMEM (FBS 2\%) was dropped again and left to stand at $4{ }^{\circ} \mathrm{C}$ for 1 day. After that, $1 \mathrm{~mL} /$ well of EGM- 2 was added and the same procedure was carried out. HUVECs were seeded at 5000 cells/well. The WST- 8 assay was performed 4 days after seeding, and the medium exchange was carried out 1 day after sowing. An amount of $2 \mathrm{~mL}$ of medium and $300 \mu \mathrm{L}$ of WST- 8 solution were mixed, and the mixture was warmed at $37^{\circ} \mathrm{C}$ for 5 minutes. Then, $230 \mu \mathrm{L} /$ well of the mixed solution was dropped and incubated in an incubator for $4 \mathrm{~h}$. Then, $30 \mu \mathrm{L}$ of $0.1 \mathrm{~N} \mathrm{HCl}$ was added. Thereafter, $100 \mu \mathrm{L}$ of the supernatant was transferred to a 96-well plate. The absorbance was measured at $450 \mathrm{~nm}$ using a microplate reader. The measurement was performed on a Microplate Photometer from Thermo Scientific (Waltham, MA, USA).

\subsubsection{H\&E Staining of the Gel after Subcutaneous Implantation in Rats}

An amount of $2 \mathrm{~mL} /$ well of MES buffer was added in each condition of Gelatin gel and ABA-Gelatin gel. After shaking overnight at room temperature, the supernatant suction was removed. Subcutaneous transplantation was performed in 9-week-old SD rats. All animal experiments were performed in accordance with the guidelines of the Ethics Committee on Animal Experiments and accepted by Kyushu University (A27-326-0, 19 Feb 2016). After 1 week, the tissue sample was taken out and immersed in $10 \%$ neutral buffered formalin solution. Tissue sections were cut out and stained with hematoxylin and eosin.

Author Contributions: H.I. and T.H. conceived and designed the experiments; D.I., T.Y., Y.-i.Y. and H.B. supplied resources; T.H., N.S., and T.I. performed the experiments; T.I. and N.S. prepared an original draft; H.I., T.I., and D.H. wrote the paper; and H.I., D.I., T.Y., Y.-i.Y., and H.B reviewed.

Funding: This research received no external funding.

Acknowledgments: The first author wishes to acknowledge the Ministry of Education, Culture, Sciences, and Sports of Mongolia for the scholarship grant.

Conflicts of Interest: The authors declare no conflict of interest. 


\section{References}

1. Nakamura, S.; Kubo, T.; Ijima, H. Heparin-conjugated gelatin as a growth factor immobilization scaffold. J. Biosci. Bioeng. 2013, 115, 562-567. [CrossRef] [PubMed]

2. Drury, J.L.; Mooney, D.J. Hydrogels for tissue engineering: Scaffold design variables and applications. Biomaterials 2003, 24, 4337-4351. [CrossRef]

3. Merion, R.M.; Ashby, V.B.; Wolfe, R.A.; Distant, D.A.; Hulbert-Shearon, T.E.; Metzger, R.A.; Ojo, A.O.; Port, F.K. Deceased-donor characteristics and the survival benefit of kidney transplantation. J. Am. Med. Assoc. 2005, 294, 2726-2733. [CrossRef] [PubMed]

4. Van Vlierberghe, S.; Dubruel, P.; Schacht, E. Biopolymer-based hydrogels as scaffolds for tissue engineering applications: A review. Biomacromolecules 2011, 12, 1387-1408. [CrossRef] [PubMed]

5. Ahmed, E.M. Hydrogel: Preparation, characterization, and applications: A review. J. Adv. Res. 2015, 6, $105-121$. [CrossRef] [PubMed]

6. Schwartz, D.A.; Petersen, B.T.; Poterucha, J.J.; Gostout, C.J. Endoscopic therapy of anastomotic bile duct strictures occurring after liver transplantation. Gastrointest. Endosc. 2000, 51, 169-174. [CrossRef]

7. Ammori, B.J.; Joseph, S.; Attia, M.; Lodge, J.P.; Beger, H.G.; Schwarz, A. Biliary strictures complicating pancreaticoduodenectomy. Int. J. Pancreatol. 2000, 28, 15-22. [CrossRef]

8. Tajeddin, E.; Sherafat, S.J.; Majidi, M.R.S.; Alebouyeh, M.; Alizadeh, A.H.M.; Zali, M.R. Association of diverse bacterial communities in human bile samples with biliary tract disorders: A survey using culture and polymerase chain reaction-denaturing gradient gel electrophoresis methods. Eur. J. Clin. Microbiol. Infect. Dis. 2016, 35, 1331-1339. [CrossRef]

9. Walsh, F. Doripenem: A new carbapenem antibiotic a review of comparative antimicrobial and bactericidal activities. Ther. Clin. Risk Manag. 2007, 3, 789-794. [PubMed]

10. Shanmugasundaram, T.; Radhakrishnan, M.; Gopikrishnan, V.; Kadirvelu, K.; Balagurunathan, R. In vitro antimicrobial and in vivo wound healing effect of actinobacterially synthesised nanoparticles of silver, gold and their alloy. RSC Adv. 2017, 7, 51729-51743. [CrossRef]

11. Queenan, A.M.; Bush, K. Carbapenemases: The Versatile $\beta$-Lactamases. Clin. Microbiol. Rev. 2007, $20,440-458$. [CrossRef] [PubMed]

12. Park, H.; Park, K. Hydrogels in Bioapplications; American Chemical Society: Washington, DC, USA, 1996; Chapter 1; pp. 2-10. [CrossRef]

13. Zhanel, G.G.; Wiebe, R.; Dilay, L.; Thomson, K.; Rubinstein, E.; Hoban, D.J.; Noreddin, A.M.; Karlowsky, J.A. Comparative review of the carbapenems-Focus on doripenem. Drugs 2007, 67, 1027-1052. [CrossRef] [PubMed]

14. Susan, J.K. Doripehem: A review of its use in the treatment of bacterial infections. Drugs 2008, 68, 2021-2067.

15. Ullah, H.; Ali, S. Classification of Anti-Bacterial Agents and Their Functions. Antibact. Agents. 2017. [CrossRef]

16. Hoare, T.R.; Kohane, D.S. Hydrogels in drug delivery: Progress and challenges. Polymer 2008, 49, $1993-2007$. [CrossRef]

17. Preparation, A. Polyvinyl Alcohol-Gelatin Patches of Salicylic Acid: Preparation, Characterization and Drug Release Studies. J. Biomater. Appl. 2006. [CrossRef]

18. Burkert, S.; Schmidt, T.; Gohs, U.; Dorschner, H.; Arndt, K.F. Cross-linking of poly (N-vinyl pyrrolidone) films by electron beam irradiation. Radiat. Phys. Chem. 2007, 76, 1324-1328. [CrossRef]

19. Gulrez, S.K.H.; Al-Assaf, S.; Phillips, G.O. Hydrogels: Methods of Preparation, Characterisation and Applications. In Progress in Molecular and Environmental Bioengineering: From Analysis and Modeling to Technology Applications; Carpi, A., Ed.; Books on Demand: Wrexham, UK, 2011. [CrossRef]

20. Conde, J.; Dias, J.T.; Grazu, V.; Moros, M.; Baptista, P.V.; de la Fuente, J.M. Revisiting 30 years of biofunctionalization and surface chemistry of inorganic nanoparticles for nanomedicine. Front. Chem. 2014, 2, 1-27. [CrossRef]

21. Sanz, V.; Conde, J.; Hernández, Y.; Baptista, P.V.; Ibarra, M.R.; de la Fuente, J.M. Effect of PEG biofunctional spacers and TAT peptide on dsRNA loading on gold nanoparticles. J. Nanopart. Res. 2012. [CrossRef]

22. Microbial Growth. Prescott's Microbiology, $9^{\text {th }}$ Edition. Available online: https://studylib.net/search/? $q=$ microbial+growth (accessed on 10 February 2019). 
23. Egawa, H.; Inomata, Y.; Uemoto, S.; Asonuma, K.; Kiuchi, T.; Fujita, S.; Hayashi, M.; Matamoros, M.A.; Itou, K.; Tanaka, K. Biliary anastomotic complications in 400 living related liver transplantations. World J. Surg. 2001, 25, 1300-1307. [CrossRef]

24. Hu, B.; Owh, C.; Chee, P.L.; Leow, W.R.; Liu, X.; Wu, Y.L.; Guo, P.; Loh, X.J.; Chen, X. Supramolecular hydrogels for antimicrobial therapy. Chem. Soc. Rev. 2018, 47, 6917-6929. [CrossRef] [PubMed]

(C) 2019 by the authors. Licensee MDPI, Basel, Switzerland. This article is an open access article distributed under the terms and conditions of the Creative Commons Attribution (CC BY) license (http://creativecommons.org/licenses/by/4.0/). 